\title{
MEASUREMENT OF VELOCITY DISTRIBUTION FOR FLOW THROUGH A BILEAFLET VALVE USING COLOR-CODED DIGITAL PARTICLE TRACKING VELOCIMETRY
}

Tienfuan Kerh

Professor, Department of Civil Engineering, National Pingtung University of Science and Technology, Pingtung, Taiwan 91207, ROC., tfkerh@mail.npust.edu.tw

Yen-Ming Chen

Engineer, TFT Photo Department, Chimei Optoelectronic, Tainan Science Park, Taiwan 744, ROC

I Tsou

Associate Professor, Department of Civil Engineering, National Pingtung University of Science and Technology, Pingtung, Taiwan 91207, ROC

Follow this and additional works at: https://jmstt.ntou.edu.tw/journal

Part of the Civil and Environmental Engineering Commons

\section{Recommended Citation}

Kerh, Tienfuan; Chen, Yen-Ming; and Tsou, I (2005) "MEASUREMENT OF VELOCITY DISTRIBUTION FOR FLOW THROUGH A BILEAFLET VALVE USING COLOR-CODED DIGITAL PARTICLE TRACKING VELOCIMETRY," Journal of Marine Science and Technology. Vol. 13: Iss. 1, Article 1.

DOI: $10.51400 / 2709-6998.2098$

Available at: https://jmstt.ntou.edu.tw/journal/vol13/iss1/1

This Research Article is brought to you for free and open access by Journal of Marine Science and Technology. It has been accepted for inclusion in Journal of Marine Science and Technology by an authorized editor of Journal of Marine Science and Technology. 
MEASUREMENT OF VELOCITY DISTRIBUTION FOR FLOW THROUGH A

BILEAFLET VALVE USING COLOR-CODED DIGITAL PARTICLE TRACKING

VELOCIMETRY

Acknowledgements

Support of this study by the National Science Council of Taiwan under project number NSC 91-2211-

E-020-008 is greatly appreciated. 


\title{
MEASUREMENT OF VELOCITY DISTRIBUTION FOR FLOW THROUGH A BILEAFLET VALVE USING COLOR-CODED DIGITAL PARTICLE TRACKING VELOCIMETRY
}

\author{
Tienfuan Kerh*, Yen-Ming Chen**, and I Tsou***
}

Key words: distribution of velocity, digital particle tracking velocimetry, semicircular bileaflet valve, opening angle.

\section{ABSTRACT}

An experimental method, the so-called color-coded Digital Particle Tracking Velocimetry system was set up in this study to investigate viscous flow through an artificial bileaflet valve with three different opening angles, $5^{\circ}, 30^{\circ}$, and $45^{\circ}$, respectively. An oscilloscope that generated stable wave speed was taken to calibrate measuring result of the equipment. Besides, a fully developed pipe flow case was used to check the reliability of experimental system, and a statistical t-test was performed to further enhance the comparison of analytical solution and measurement. From the details of randomly distributed velocities, interpolated velocity components, and velocity profiles for the studied flow case, it could be observed that three jetlike flows through upper, central and lower parts of the two leaflets occurred in this flow configuration. Due to the effect of shear layer at wall boundary and at valve wake, the jet-like flows interfered with each other, and caused that the larger the opening angle, the stronger the recirculation region at downstream of valve structure. As a result, the velocity distributions exhibited more oscillating phenomenon in both axial and radius directions for larger opening angle case. From error analysis, although these measured results might involve some uncertainties and incompleteness, they might provide basic flow characteristics in this type of flow case and relative applications.

\section{INTRODUCTION}

Flow problems in general can be studied by three approaches, which are theoretical method, computer simulation and experimental measurement. As the fluid motion usually involves a very complicated nonlinear behavior, which is not easy to get an analytical solution,

Paper Submitted 10/02/03, Accepted 04/29/04. Author for Correspondence: Tienfuan Kerh. E-mail: tfkerh@mail.npust.edu.tw.

*Professor, Department of Civil Engineering, National Pingtung University of Science and Technology, Pingtung, Taiwan 91207, ROC.

**Engineer, TFT Photo Department, Chimei Optoelectronic, Tainan Science Park, Taiwan 744, ROC.

***Associate Professor, Department of Civil Engineering, National Pingtung University of Science and Technology, Pingtung, Taiwan 91207, ROC. and that may limit the theoretical method only applicable for some simple flow cases (e.g. colliding flow, Couette motion; $[12,13])$. In contrast, the computer simulation using numerical methods such as finite element method, finite difference method and finite volume method, can obtain detailed flow fields to describe fluid motion in many engineering applications once the boundary conditions are properly defined. However, the computational result requires available experimental data to verify its accuracy and to check its reliability $[14,15]$. Hence, the experimental result may not as complete as numerical simulation, but the measurement may become valuable and a requirement for researching in this important area, either from theoretical or from practical point of view.

Distribution of velocity is one of the key elements to analyze flow field characteristics in different geometrical flow domains. There are many instruments available for measuring flow velocity, such as the Laser Doppler Velocimetry (LDV) or Laser Doppler Anemometry (LDA), which can obtain a very high precision result, but it may be expensive to build up the system and may take a long time to obtain data point by point in the flow domain $[6,19]$. Another popular measuring example is the Particle Image Velocimetry (PIV), which is cheaper and can take flow images directly from digital camera, it requires a large amount of memory to store the images and the post-analysis may be also time consumption [1, 9, 17]. However, these drawbacks are no longer existent as more advanced technology of hardware and software have been developed in the recent years. Alternatively, for the sake of economical concern, the Digital Particle Tracking Velocimetry (DPTV, [27, 28]) based on the principle of PTV [10, 18, 22] may consider as an applicable tool, which is able to obtain the distribution of velocity by interpolation for the entire flow domain in a short time without too much computer storage. Thus, in the present study, a colorcoded DPTV experimental system is set up to measure 
flow velocity profiles at the downstream of a semicircular bileaflet valve situated in a closed conduit.

The configuration of bileaflet valve may be frequently found in the field of biomedical engineering. For instance, due to the advantages of low profile and less side effects reported in the real world, the most popular mechanical valves used in recent years such as St. Jude, Carbomedics and Edwards-Duromedics, mainly consist of a set of bileaflet. Without a doubt, there are many reports including numerical computations and experiments have been published in this crucial topic $[2,6,7,16,23,24]$. However, the application of using a color-coded DPTV to investigate this flow problem is rarely seen and still opened for latter researchers. Therefore, the purpose of this study is mainly to set up a DPTV experimental system to measure flow field, in particular, the distribution of velocity for flow through a semicircular bileaflet device with different open angles in a closed conduit.

The contents of this study include at the beginning, an oscilloscope capable of creating a highlighted point with stable wave speed, 5.2 and $10.4 \mathrm{~cm} / \mathrm{s}$, respectively, is taken to calibrate the measuring equipments. Then, the comparisons of analytical solutions with measurements in a fully developed laminar pipe flow at Reynolds number equals to 1500 is employed to check the reliability of experimental system. Finally, the measurement is focused on the case of semicircular bileaflet valve existed in the flow domain. A steady flow through three different bileaflet opening angels, $5^{\circ}, 30^{\circ}$, and $45^{\circ}$, respectively, is examined on the downstream region of the valve system. The details of randomly distributed velocity, interpolated velocity in different components, and velocity profile at selected sections are presented for the three opening angles. The errors or uncertainties are described and estimated according to various sources in the measuring system. The measured results may provide an insight of this flow case and may provide useful information for the relevant engineering applications.

\section{EXPERIMENTAL SETUP OF THE DPTV SYSTEM}

To obtain the distribution of velocity in flow domain, the experimental instruments must be properly arranged. As sketched in Figure 1, the present experimental measurement setup is a recursive piping system, which includes the following major items: (1) An inlet and an outlet water storage tanks of dimensions $47.5 \times$ $47.5 \times 100 \mathrm{~cm}^{3}$ and $38 \times 38 \times 50 \mathrm{~cm}^{3}$, respectively. A spill hole at the upper region of inlet tank is worked to keep the water level at the same height. (2) A pump at the outlet tank that can provide 90 liters water per minute. (3) A water temperature controller for preventing temperature variation during flow measurement. (4) Two straight circular acrylic pipes, the first section has $2.8 \mathrm{~cm}, 2.5 \mathrm{~cm}$ and $2 \mathrm{~m}$ in outer diameter, inner diameter and total length, respectively. The second section has $6.0 \mathrm{~cm}, 5.6 \mathrm{~cm}$ and $4 \mathrm{~m}$ in outer diameter, inner diameter

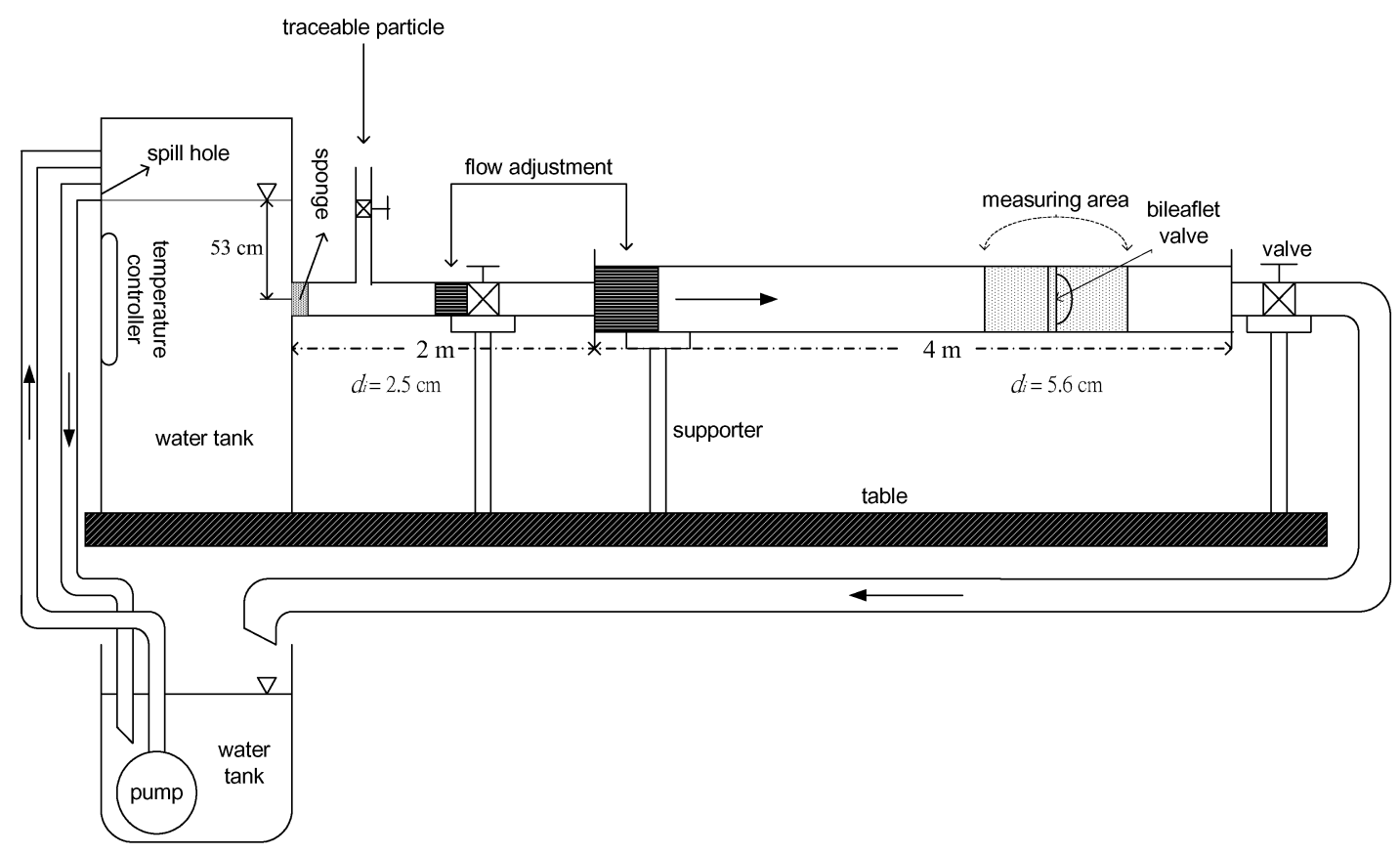

Fig. 1. Sketch of major items in experimental recursive piping system. 
and total length, respectively. In between, a bundle of straw is inserted as buffer zone for adjustment of flowing condition, each straw has $7 \mathrm{~cm}$ in length and $0.5 \mathrm{~cm}$ in diameter. (5) Other components such as valves, sponges and supporters are added for controlling flow rates and putting fluorescent particles.

In the measuring area, a detailed connection of instruments is displayed in Figure 2. Note that a $20 \mathrm{~mW}$ He-Ne laser beam by Uniphase is taken as the illuminating source, and a beam splitter prism glass bar with convex lens is used to form a light sheet in this investigation region. In addition, manufactured by Duke Scientific Corporation, the traceable polystyrene microspheres of each have $50.4 \pm 1.0 \mu \mathrm{m}$ in diameter and 1.05 $\mathrm{kg} / \mathrm{m}^{3}$ in density are taken as the fluorescent seeding particles for tracking the water flow. For capturing flow images, the Dalsa-Cad1-0256 charge coupled device (CCD) camera, with resolution $256 \times 256$ pixels is used with Coreco Oculus-F/64 frame grabber board in personal computer $[5,20,21]$. This device contains arithmetic logic unit (ALU), input look up table (ILUT), output look up table (OLUT) and video random access memory (VRAM), which is capable of transferring images into monitor and film recorder. Furthermore, the DPTV software and Tecplot software [4, 11, 25] are applied to analyze the measuring results and post-plots by their multi-functions.

The principle of particle tracking technique is basically by taking the trajectory of moving particle in a frame image, which records both of length and time

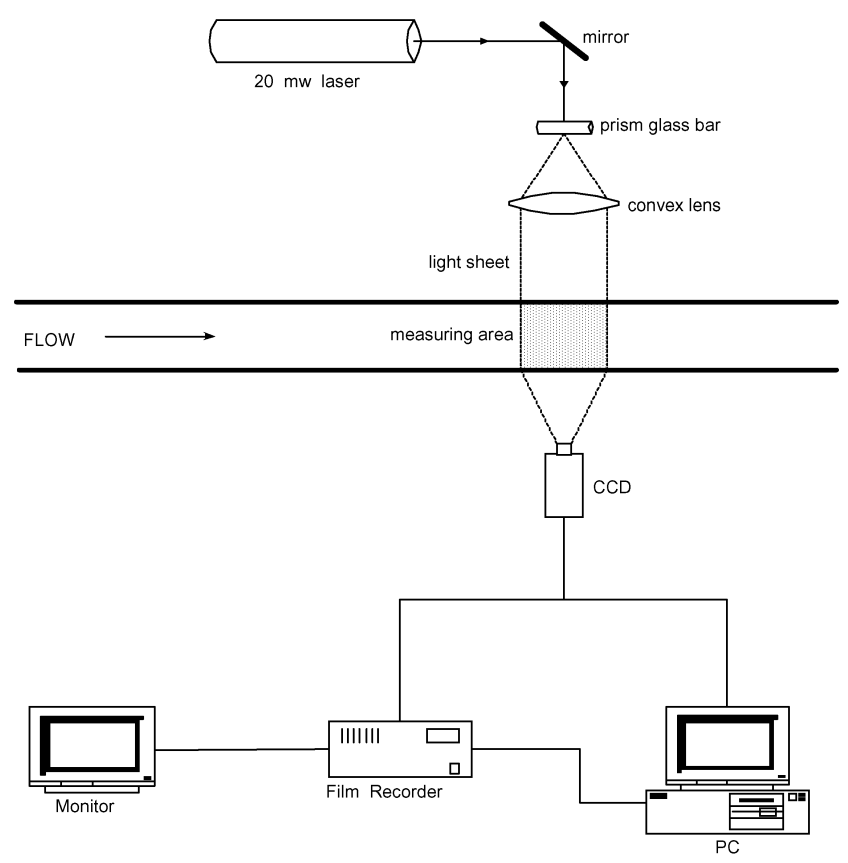

Fig. 2. Instrumental connection details in the measuring area. parameters to calculate the flow velocity. The present DPTV system uses Computar MLH-10X variable focus lens with zoom $0.084 \mathrm{X}-0.84 \mathrm{X}$ and diaphragm F5.6 in CCD camera. This instrument is able to measure the flow velocity as low as $0.41 \mathrm{~cm} / \mathrm{s}$ within a $5.5 \times 5.5 \mathrm{~cm}^{2}$ frame, which is suitable for the present flow configuration and is sufficient for laminar flow measurement. Once the flow images are taken from the CCD camera continuously, an overlay image with four gray levels from sequential binary images is gained by using ILUT and ALU. Then, by defining the four gray levels with the use of OLUT, a color-coded image (i.e. red, yellow, green and blue colors represent four different gray levels) is obtained to view the result more clearly. After thinning treatment to the structure of color-coded image, the simplified image information can be easily stored in computer for further analysis.

\section{CALIBRATION AND VERIFICATION OF THE EXPERIMENTAL SYSTEM}

There are many factors may affect the experimental result and cause an error during the entire measuring process. To reduce the occurrence of errors in experiment, some efforts may be made in this study. For example, the estimation of water temperature will arise about $4.9 \times 10^{-4 \circ} \mathrm{C}$ from the $20 \mathrm{~mW}$ laser light, which is minor and a temperature controller is used to prevent its variation. Another example is the use of polystyrene fluorescent particle, which is expensive but much better than the common aluminum particle. The polystyrene particles may uniformly distribute in fluid flow, and the seed has a very small relative velocity $\left(1.9 \times 10^{-2} \mathrm{~cm} / \mathrm{s}\right.$ estimated) by comparing with flow velocity to reduce the drag force effect. Additional examples such as using a scaled ruler to revise the photo incline angle effect, shortening the exposure time as possible to decrease the flow fluctuation effect, choosing a suitable interpolation method and reducing inlet flow velocity to reduce the recirculation effects. Also, one concern is that the present method uses head-tail connected straight line to reconstruct the velocity fields, the error may be generated due to the curve surface of pipe and also due to the difference of refraction coefficients in light transport media which including water, acrylic pipe, air and CCD lens. The detailed analysis of these effects can be a very complicated work, but a simplified way to estimate the error may be shown as the following auxiliary figure and equation.

$$
\varepsilon=\frac{R \theta-L}{R \theta} \times 100 \%
$$

In Figure 3 and equation (1), $R$ is the curvature radius, $\theta$ is the relative angle, $L$ is the length of calcu- 
lated velocity, $R \theta$ is the real length of curve line, and $\varepsilon$ is the relative error. The error is small for a smoother flow field area, but it will become significant for a large flow recirculation zone, which may limit the accuracy of a large Reynolds number flow condition and a large bileaflet open angle flow case. The correction of measured velocity field may be made according to the above equation, but it may become a tedious task for a randomly distributed velocity field.

Nevertheless, no matter what reason to cause the error, it is important to calibrate the experimental setup using available methods. In this study, an oscilloscope capable of creating a highlighted point with stable wave speed is taken as a tool to check the measuring equipments. The applied model China Tech D50102 oscilloscope can provide single point wave in a length of $10.4 \mathrm{~cm}$ region, with periods of 2.0 and 1.0

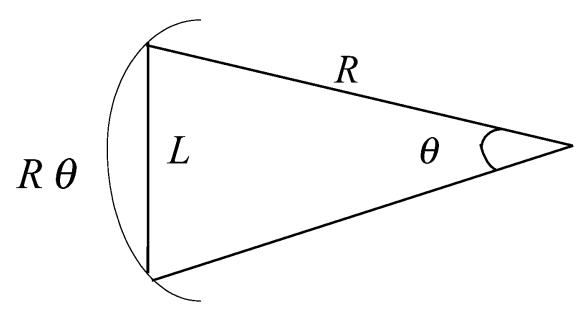

Fig. 3. Physical relationship between curve line and straight line.

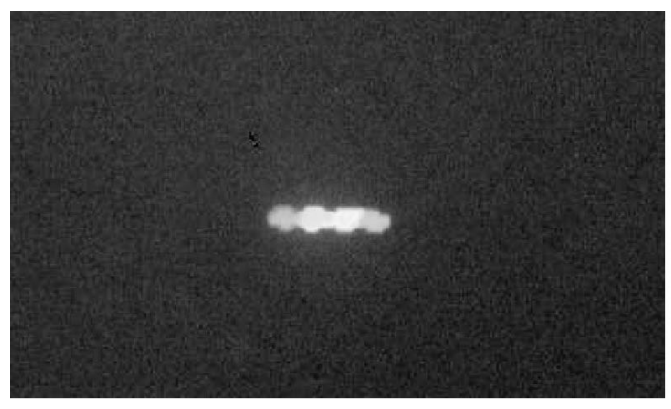

(a) $5.2 \mathrm{~cm} / \mathrm{sec}$ (original)

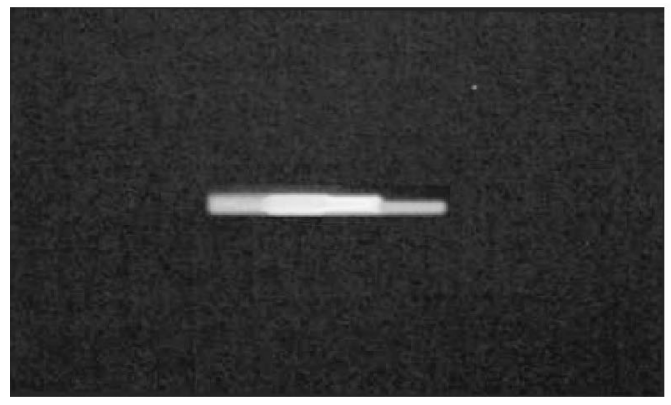

(b) $10.4 \mathrm{~cm} / \mathrm{sec}$ (original) seconds, which are equivalent to 5.2 and $10.4 \mathrm{~cm} / \mathrm{s}$ of wave speeds, respectively. Shown in Figure 4 is the original and thinning tracer for the two different wave speeds grabbed by the color-coded DPTV system. Because a small part of line segments at the head and the end of the four-color tracer may be cut after thinning, thus only two portions in the middle of tracer are taken for velocity calculation, and the equations are as follows:

$$
\begin{aligned}
& u=\left(X / N_{x}\right)\left(N_{2 x}-N_{1 x}\right) / \Delta T \\
& v=\left(Y / N_{y}\right)\left(N_{2 y}-N_{1 y}\right) / \Delta T
\end{aligned}
$$

where $u$ and $v$ are the velocities in $\mathrm{x}$ and $\mathrm{y}$ directions, respectively; $X$ and $Y$ are the image actual lengths in $x$ and $y$ directions, respectively; $N_{x}$ and $N_{y}$ are the numbers of image pixels in $x$ and $y$ directions, respectively; $N_{1 x}, N_{1 y}$ are the initial image coordinates at the second line segment; $N_{2 x}, N_{2 y}$ are the end image coordinates at the third line segment; and $\Delta T$ is the time interval. As different frame size may result in different resolution, which will have an influence on the measured velocity. The present experimental system is tried to perform measurement with different frame areas, and a relatively better frame size $5.5 \times 5.5 \mathrm{~cm}^{2}$ with 3 images for each color segment is chosen for fitting in flow cases studied herein.

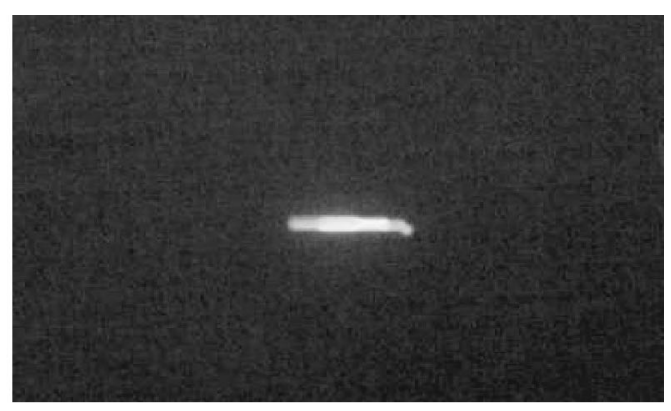

(after thinning)

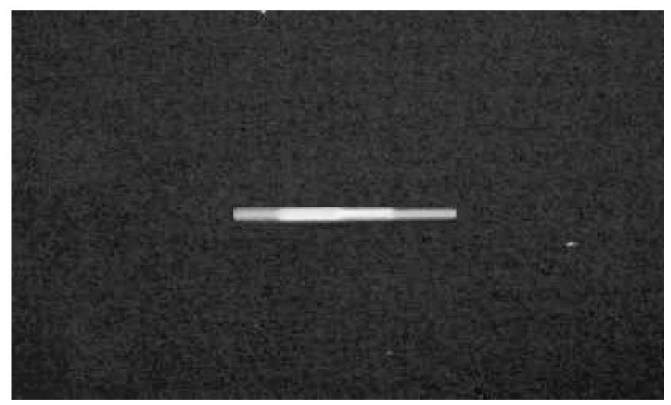

(after thinning)

Fig. 4. Original traces and color segments after thinning for different particle speeds. 
To further check the reliability of measurement system, in the next is the comparison of fully developed flow in a conduit. As displayed in Figure 5 the experimental result is compared with analytical solution for Reynolds number equal to 1500 . For a laminar circular pipe flow, the analytical solution can be theoretical obtained from the velocity equation according to Newton's second law in a control volume [29]. The Reynolds number is defined as $\operatorname{Re}=V_{a v g} D / v$, where $v$ is the kinetic viscosity of fluid, and $D$ is the pipe diameter. Here the averaged flow velocity $V_{\text {avg }}$ is calculated from flow rate and pipe cross-section area at the outlet of experimental system. Note that the randomly flow velocity is a superposition result from 30 times experimental measurements. Then, by taking 10 profiles in $x$ direction and 70 profiles in $y$-direction, with the use of inverse distance weighting method, the interpolated velocity profiles are obtained, and a profile is used to make comparison with analytical solution. It can be seen that the experiments are in reasonable agreements with analytical solutions for this flow case. To further enhance the above statement, a statistical $t$-test is per-

(a)

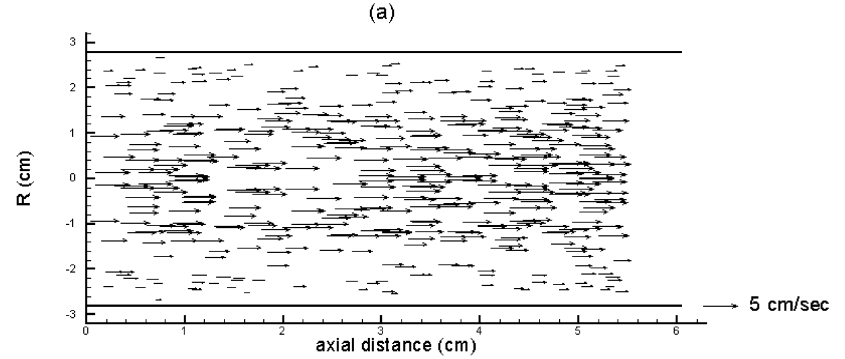

(b)

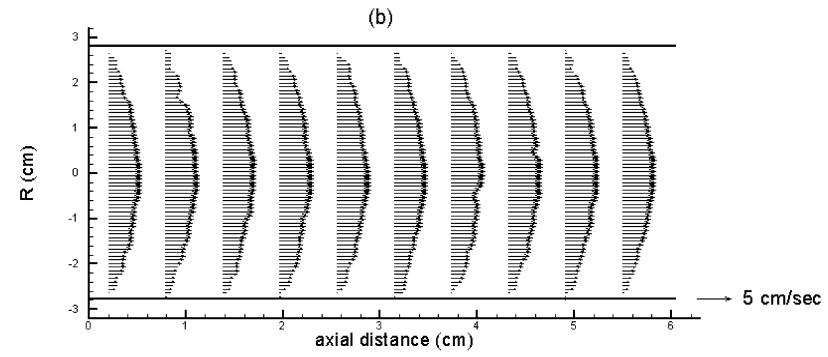

(c)

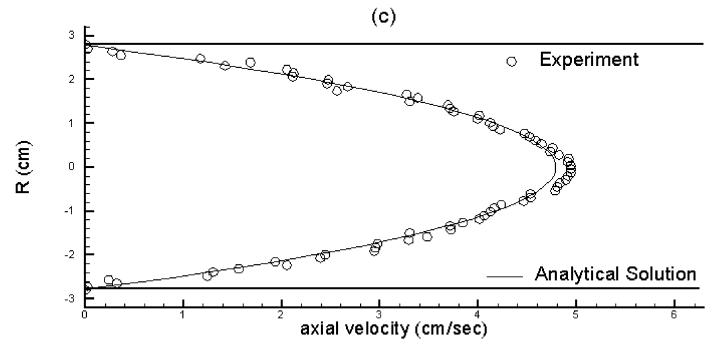

Fig. 5. Distribution of velocity: (a) randomly velocity, (b) interpolated velocity, and (c) comparison of experimental result with analytical solution $(\operatorname{Re}=1500,[29])$. formed for the case, and the result is $t=-1.303$. This value passes the test as it complies with the acceptance interval $-1.995<t<1.995$, under 69 degree of freedoms and $5 \%$ significance level. This comparison provides us confidence of using the present color-coded DPTV system, and the same technique will be applied for examining more complicated flow case in the next section.

\section{DISTRIBUTION OF VELOCITY FOR FLOW THROUGH A BILEAFLET VALVE}

Now by considering an artificial bileaflet valve is placed into the circular pipe in the investigating area, and a detailed drawing of the valve is shown in Figure 6 . The opening angle of leaflet $(\alpha)$ is able to adjust by the screws, and there are three opening angles, $5^{\circ}, 30^{\circ}$ and $45^{\circ}$ are examined in this study. This bileaflet valve has a semicircular shape, with $2 \mathrm{~cm}$ in radius and $0.3 \mathrm{~cm}$ in thickness, which is connected to a $0.9 \mathrm{~cm}$ wide ring with $0.4 \mathrm{~cm}$ in thickness. Due to the pipe boundary is supported by the artificial valve, the diameter of circular pipe is constricted from $5.6 \mathrm{~cm}$ to $4.8 \mathrm{~cm}$, in addition to the effect of two leaflets, the fluid motion may expect to become very complicated at the downstream. To prevent too much errors may occur in measurement for the high recirculating flow problem, a moderate Reynolds number $\operatorname{Re}=1196$, as defined in previous section, is measured in this flow case.

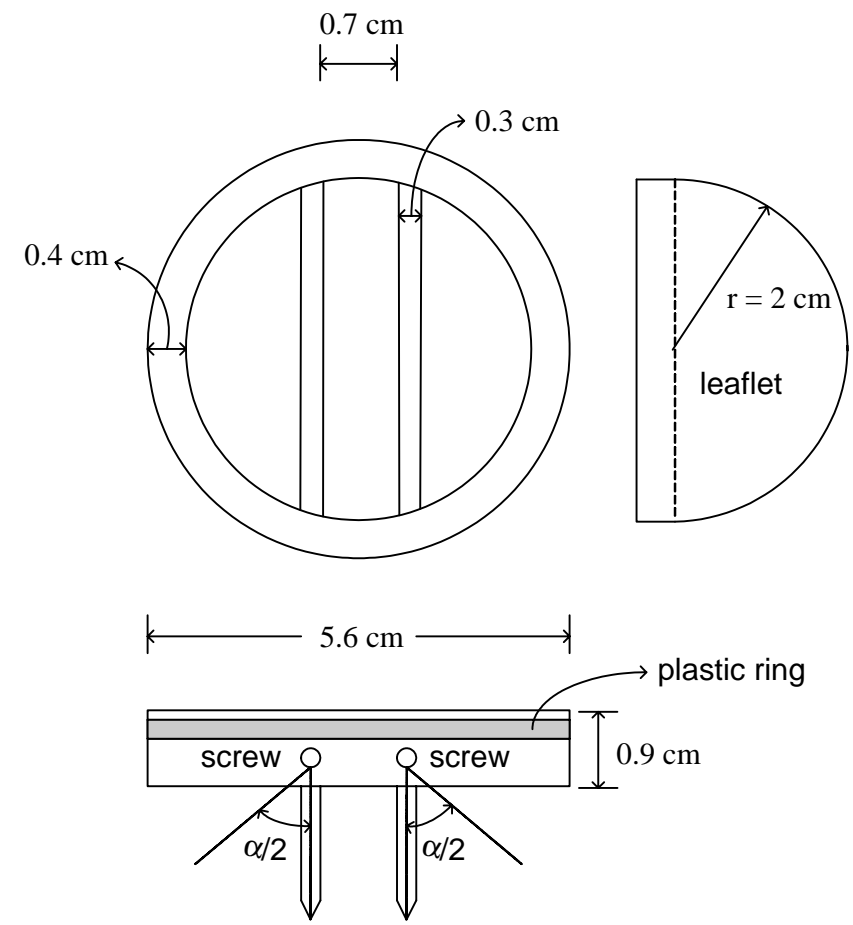

Fig. 6. Detailed sketch of the artificial bileaflet valve. 
The distributions of velocity are shown in Figures 7,8 , and 9 for opening angles equal to $\alpha=5^{\circ}, 30^{\circ}$, and $45^{\circ}$, respectively. In these figures, plot (a) presents the randomly distributed velocity, which is obtained from a superposition of 30 times measurements at the same flow condition; plot (b) denotes the interpolated velocity; plot (c) is the interpolated velocity in axial-component; and plot (d) is the interpolated velocity in radialcomponent. It is noted that the two leaflets and the opening angles are shown as a reference, which is not scaled to fit in velocity vectors in each plot. Also note
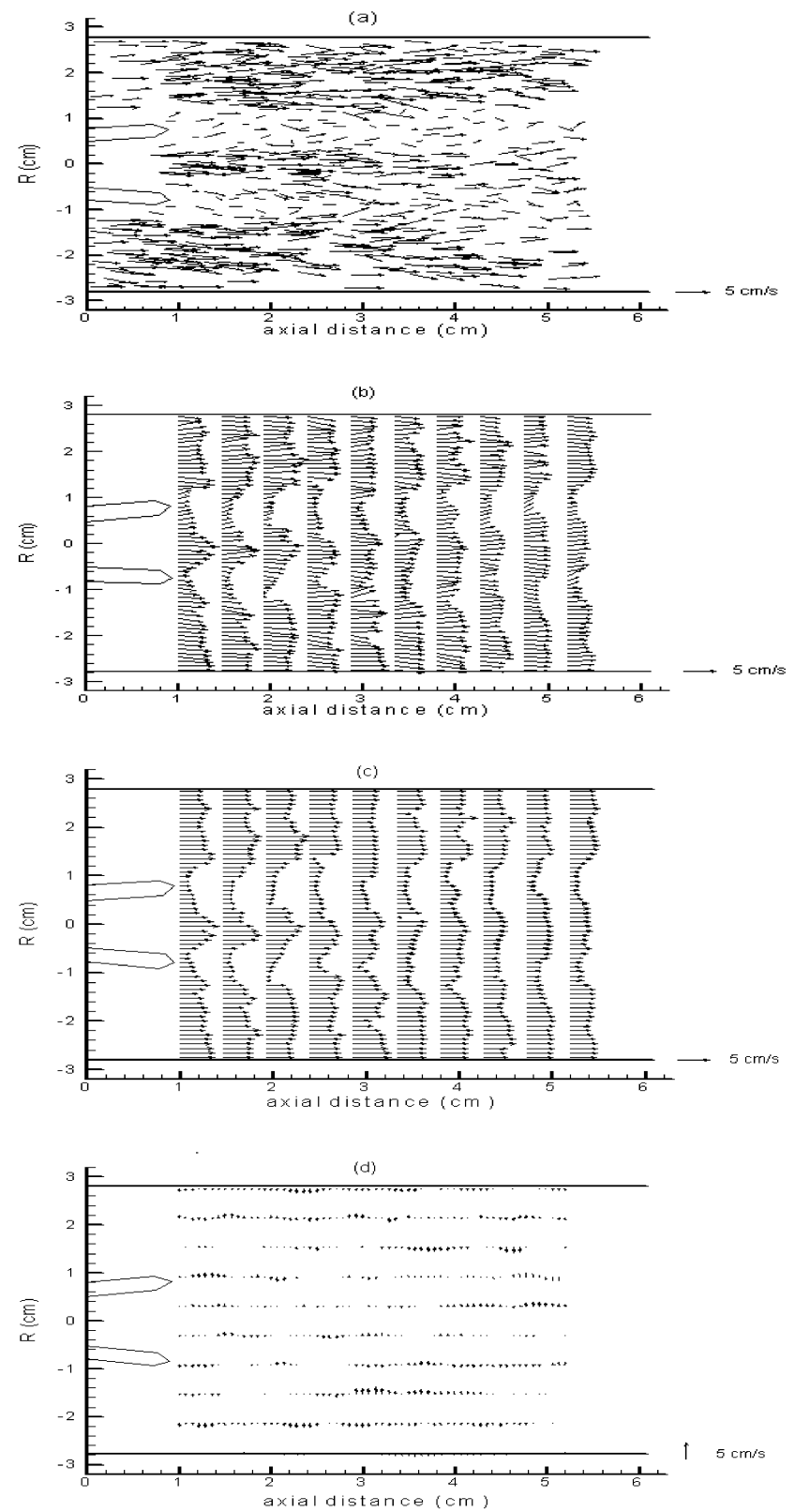

Fig. 7. Distribution of velocity: (a) randomly distributed velocity, (b) interpolated velocity, (c) velocity in axial-direction, and (d) velocity in radial-direction $\left(\alpha=5^{\circ}, \operatorname{Re}=1196\right)$. that in plot (a) of these figures, because the laser beam light source can not go through the valve structure, and the velocity at valve region can not be captured completely by the CCD camera, so that the continuity equation is not held near this region. By checking the mass conservation of each cross section, the variations may range about up to $3.6 \%, 4.2 \%$, and $40.6 \%$ for the opening angles equal to $\alpha=5^{\circ}, 30^{\circ}$, and $45^{\circ}$, respectively. That is, the measuring results exhibit less accuracy or may even have unreliable measurements due to highly disturbed flow situation in the highest opening angle flow
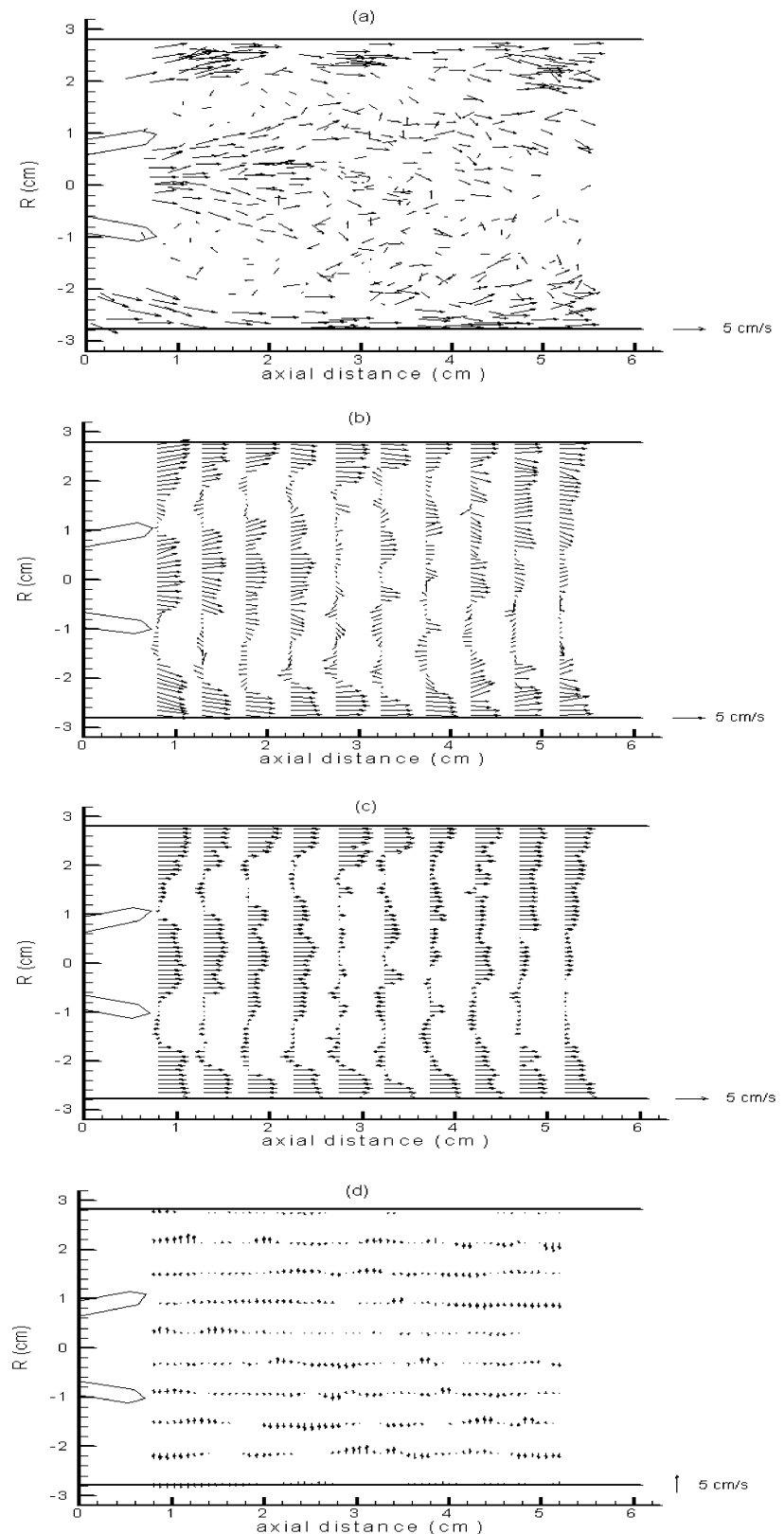

Fig. 8. Distribution of velocity: (a) randomly distributed velocity, (b) interpolated velocity, (c) velocity in axial-direction, and (d) velocity in radial-direction $\left(\alpha=30^{\circ}, \mathbf{R e}=1196\right)$. 
case. However for all cases, this principle has a better shape for velocity farer at downstream of the valve body despite the existence of some measuring errors.

From all these plots, it can be found that at opening angle $\alpha=5^{\circ}$, because the two leaflets are nearly parallel to each other, the flow at downstream does not disturb too much by the valve body, thus the recirculation area is not found from the randomly distribution of velocity. In this situation, there exists no negative interpolated velocity component in axial direction, and small value is found for velocity component in radial direction. But
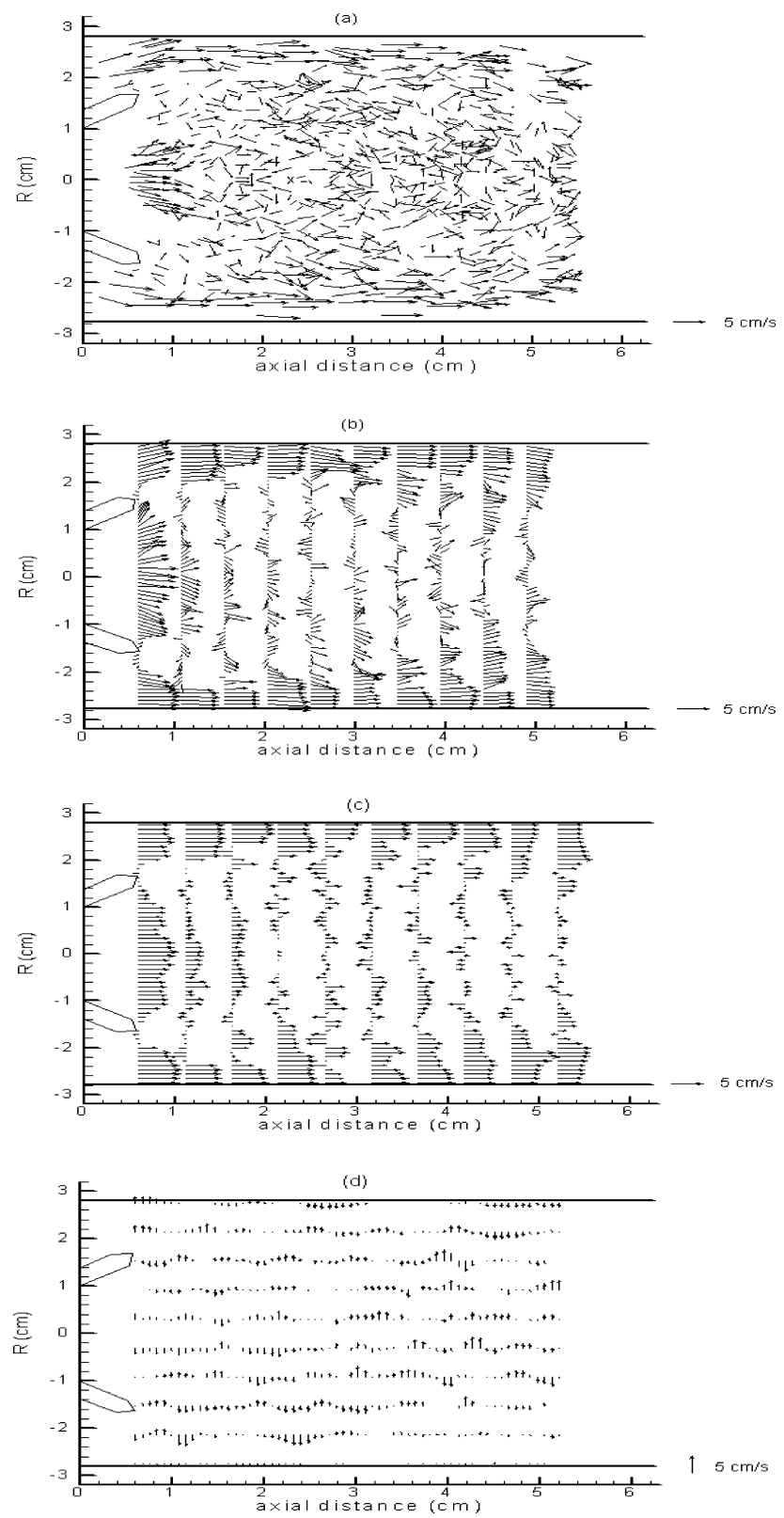

Fig. 9. Distribution of velocity: (a) randomly distributed velocity, (b) interpolated velocity, (c) velocity in axial-direction, and (d) velocity in radial-direction $\left(\alpha=45^{\circ}, \operatorname{Re}=1196\right)$. as the opening angle becomes $\alpha=30^{\circ}$, the recirculation region is easy to observe at downstream of the leaflet due to flow separation. This wake flow effect results in the negative velocity components in both axial and radial directions which are getting larger by comparing with previous case. When the opening angle reaches $\alpha$ $=45^{\circ}$, the flow is disturbed very significantly by the valve structure, and the recirculation zone is the strongest among these three opening angles. Thus more oscillating negative velocity components in both axial and radial directions are found from the plots. In all cases, it can be seen that there are three jet-like flows through upper, central and lower parts of the two leaflets. Because of flow squeezing effect for the two leaflets with boundary walls, there may exist a switching of flow field, but it is difficult to observe this phenomenon as it will change with time. From the present steady limited sample measurements, a slightly oscillation result may be found from velocity profiles at radial direction as shown in Figure 7(d) to Figure 9(d) for three opening angles. It can be seen that positive and negative velocities of radial direction in turn along the axial distance component, and which become more significant for the larger opening angle case. The detail quantity of these velocities may be taken from a profile of the plots, but there are small values by comparing with velocities in axial-component. This oscillation behavior may cause the jet flows near upper wall and lower wall regions to grow their strength and mixing with the central jet flow, which may cause chaotic flow fields and make it more difficult to measure velocity field in the larger opening angle flow case.

From biomedical engineering standpoint, the recirculation zone will cause thrombus formation if an artificial valve is implanted in a blood vessel. Furthermore, since the definition of wall shear stress is proportional to the gradient of velocity near wall boundary, hence the occurrence of jet flows near upper and lower walls will damage the blood cells along the vessel boundary after a sufficiently long time action. These side effects of using an artificial valve must be carefully concerned for the persons working in this area. Actually, these negative impacts are inevitable as the artificial valve is in general made by rigid material, which may never resemble the elastic feature of a real heart valve. An elastic type of artificial valve may be designed in today, but it is easy to damage and suffers from failure for using repeatedly after a period of time. As soon as the technology in material science is improved to overcome weakness of an elastic valve, the behavior of flow separation at valve tail may be reduced to decrease the negative side effects.

To further understand the degree of velocity field affected by the bileaflet opening angle, three interpo- 
lated axial velocity profiles at valve downstream $2.2 \mathrm{~cm}$ (section 1), $3.2 \mathrm{~cm}$ (section 2) and $4.2 \mathrm{~cm}$ (section 3), are taken for analysis. Note that these interpolated velocity profiles are chosen to facilitate the comparison, which are not shown in previous velocity distributions. Presented in Figure 10, 11, and 12 are the results for three opening angles at these three sections. Because the chosen sections are somewhat a little bit away from the valve body, it can be observed that there is not too much difference for the cases of $\alpha=5^{\circ}$ and $\alpha=30^{\circ}$, but there does exist more velocity variations and more negative velocities for the case of $\alpha=45^{\circ}$. Theoretically, these velocity profiles should be in a symmetrical shape as long as every item of measurement is under controlled. However, the shape of two leaflets may never be produced really symmetry, the valve structure may not be inserted precisely in the circular pipe, and the angle between two leaflets may not be opened exactly. In addition, other experimental components such as screws, plastic rings and bubbles, all may have an influence on the flow measurement.

In this study, the averaged measuring results are yielded by 30 experimental measurements at the same condition, which may not be sufficiently to generate an accurate result for fitting in the theoretical concept. But, the 30 sample results fulfill the minimum require-

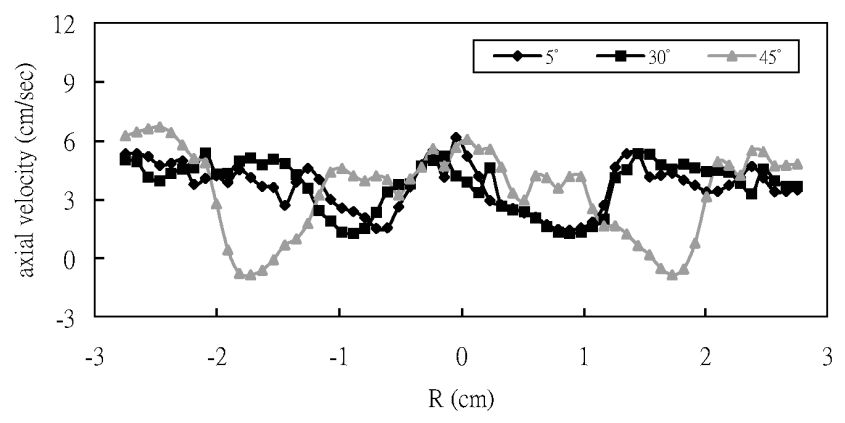

Fig. 10. Velocity profiles at section 1 for three opening angles $(\mathrm{Re}=$ 1196).

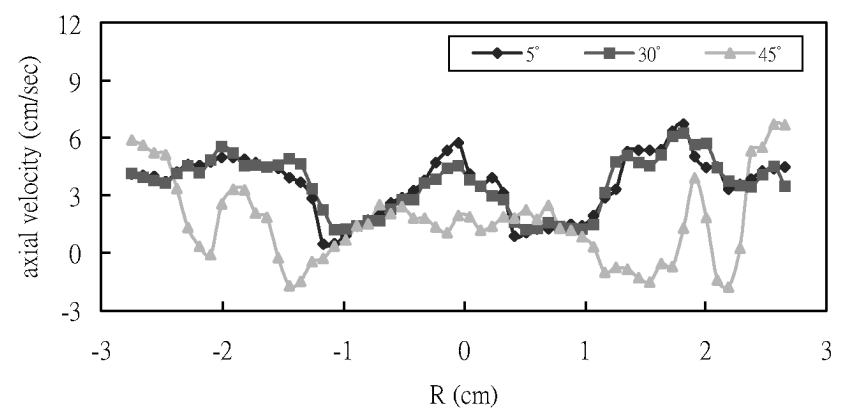

Fig. 11. Velocity profiles at section 2 for three opening angles $(\operatorname{Re}=$ 1196). ment from a statistical sense. Furthermore, from the generation of error sources as described in section 3 , the errors or uncertainties in the present measuring results may be estimated by the following items: (1) the error from thermal effect is neglected because it is too small and a controller is used to keep a constant water temperature; (2) the relative velocity $1.9 \times 10^{-2} \mathrm{~cm} / \mathrm{s}$ of polystyrene particles may produce $0.18 \%-0.37 \%$ errors if the maximum flow velocities range from $5.2 \mathrm{~cm} / \mathrm{s}$ to $10.4 \mathrm{~cm} / \mathrm{s}$ are used for comparison; (3) by using the calibration result from an oscilloscope with two different wave speeds, the image resolution errors have $1.07 \%-4.96 \%$ based upon $5.5 \times 5.5 \mathrm{~cm}^{2}$ frame size with 3 images for each color segment; (4) an experimental result of rotating disc may be applied to estimate the errors between curve line and straight line segments [3], and the interpolated values are $4.24 \%-5.29 \%$ for both velocities; (5) the inclined angle between $\mathrm{CCD}$ and measuring face is controlled within the range of $1^{\circ}-5^{\circ}$ in general, and thus the errors are about $0.03 \%-0.38 \%$ [26]; (6) the image distortion caused by light refraction is about under two pixels which may produce $0.1 \%-$ $0.8 \%$ errors [26]. Therefore, the present experimental system may generate $5.62 \%-11.80 \%$ estimated errors in total, and the root mean square errors (RMSE) are $4.38 \%-7.31 \%$ for both reference flow velocities. These uncertainties do affect the accuracy of measurement, but the characteristics of resulting flow field may still have a technical reference merit. Surely, much more measurements such as over 1000 times at the same flow condition may be performed for increasing the accuracy, and a flow visualization may be included to enhance the results, but the present budget-limit experimental system has its limitation, which is out of capability to generate a precise result. In addition, the opening angle of valve structure may be adjusted by try-and-error, or even by taking only one half of measured velocity profile as the basis to produce a fully velocity profile using mapping technique to satisfy the symmetric behavior. Whereas, these modifications may have a controversy as the measuring conditions can never be

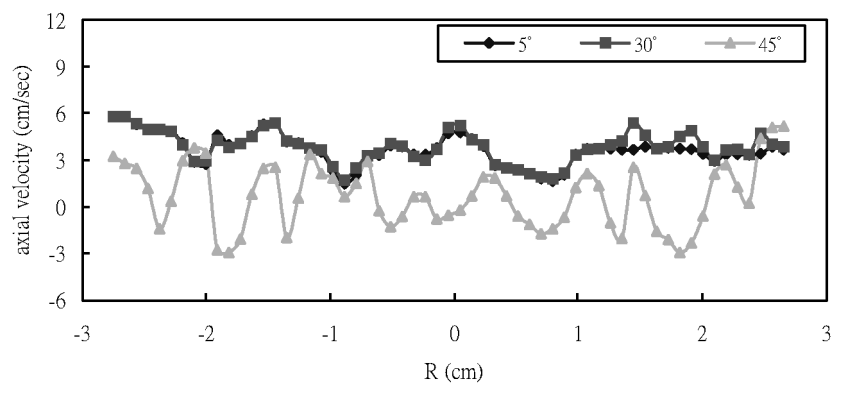

Fig. 12. Velocity profiles at section 3 for three opening angles $(\operatorname{Re}=$ 1196). 
perfect in practice. Anyway, with a limited resource of experimental system, the present measuring results do involve some errors and incompleteness, but they may still reflect some of important flow patterns, which provide a basic insight of flow characteristics in this type of flow case. Some other ways may be applied to improve the accuracy of measurement, such as by using a higher power of laser beam to capture the traces more easily, and by installing more video random access memory to increase the image resolution, but these are out of the present experimental resources. Although the present color-coded DPTV measurement system may not as good as a LDV system, it does provide a minimum requirement for flow measurement, and the obtaining results may have a suitable value for reference in engineering applications.

\section{SUMMARY AND CONCLUSION}

Due to cost inexpensive and the capacity of obtaining the distribution of velocity by interpolation for the entire flow domain in a short time without too much computer storage, in this paper, a color-coded Digital Particle Tracking Velocimetry experimental system has been set up to investigate viscous flow through an artificial bileaflet valve with different opening angles. The details of randomly distributed velocities, interpolated velocity components, and velocity profiles at the chosen sections have been presented and illustrated for the different flow cases.

At the beginning of this study, the experimental system has been calibrated by using an oscilloscope that is capable of creating two optional stable wave speeds $(5.2$ and $10.4 \mathrm{~cm} / \mathrm{s})$. And a suitable frame size has been decided in measurement for the present flow configuration. In the next, the measurements have been compared with analytical solutions in a case of fully developed pipe flow. A Reynolds number (1500) has been studied and the statistical $t$-test result has proved the reliability of the present experimental method. Finally, the measurement has been focused on the case of semicircular bileaflet valve included in the flow domain. Three different bileaflet opening angels $\left(5^{\circ}\right.$, $30^{\circ}$ and $45^{\circ}$ ) have been examined on the downstream region of the valve system.

The measured results have shown that there existed three jet-like flows through upper, central and lower parts of the two leaflets. Because of flow squeezing effect for the two leaflets with boundary walls, the jet flows near upper wall and lower wall regions have grown their strength and mixing with the central jet flow. This flow separation phenomenon has caused the larger the opening angle, the stronger the recirculation area under the flow conditions studied herein. In consequence, the wake flow effect has resulted in more oscillating negative velocity components in both axial and radial directions for larger opening angle case. The measuring results obtained from the present budgetlimit experimental system might involve some errors and incompleteness (totally estimation errors $5.62 \%-$ $11.80 \%$, and the root mean square errors $4.38 \%-$ $7.31 \%$ ), but they might have reflected some of important basic flow patterns. Although other measurement equipment or more measurements at the same flow condition might be employed for improving the accuracy, it might cost expensive and time consumption. Thus for economical concern, the present experimental results might moderately provide an insight of flow characteristics in this flow type, and might provide useful information for the relative engineering applications.

\section{ACKNOWLEDGEMENTS}

Support of this study by the National Science Council of Taiwan under project number NSC 91-2211E-020-008 is greatly appreciated.

\section{REFERENCES}

1. Adrian, R.J., "Particle-Image Techniques for Experimental Fluid Mechanics," Annu. Rev. Fluid Mech. Vol. 23, pp. 261-304 (1991).

2. Carl, E.E.K., Eugene, H.B., and Tjark, E., "Thromboembolism and Mechanical Heart Valves: A Randomized Study Revisited," Ann. Thorac. Surg., Vol. 66, pp. 101 -107 (1998).

3. Chen, R.S., "Measurement of Flow Velocity Distribution in a Orifice by Particle Image Tracking Velocimetry," Master Thesis, Department of Mechanical Engineering, National Central University (1996).

4. Chen, T.S., "DPTV Operation Manual," Version 1.120, Taiwan (1995).

5. Dalsa Cad1 Camera, "Interface with F/64 Board," Dalsa Inc., USA (1996).

6. De Hart, J., Peters, G.W.M., Schreurs, P.J.G., and Baaijens, F.P.T., "A Two-Dimensional Fluid-Structure Interaction Model of the Aortic Valve," J. Biomech., Vol. 33, pp. 1079-1088 (2000).

7. Ellis, J.T., Hearly, T.M., Fontaine, A.A., Weston, M.W., Jarret, C.A., Saxena, R., and Yoganathan, A.P., "An in Vitro Investigation of the Retrograde Flow Fields of Two Bileaflet Mechanical Heart Valves," J. Heart Valve Dis., Vol. 5, pp. 600-606 (1996).

8. Fatemi, R. and Chandran, K.B., "An In Vitro Comparative Study of St. Jude Medical and Edwards- Duromedics Bileaflet Valves Using Laser Anemometry," J. Biomech. Eng., Vol. 111, pp. 298-302 (1998). 
9. Hassan, Y.A., Philip, O.G., and Schmidl, W.D., "TwoPhase Velocity Measurements Around Cylinders Using Particle Image Velocimetry," Adv. Multiph. Flow, pp. 759-769 (1995).

10. Hering, F., Leue, C., Wierzimok, D., and Jahne, B., "Particle Tracking Velocimetry Beneath Water Waves. Part 1: Visualization and Tracking Algorithms," Exp. Fluids, Vol. 23, pp. 472-482 (1997).

11. Hsu, V.C., "F64 Driver for DPTV," Nowa Co. Ltd., Taiwan (1996).

12. Kerh, T., Chung, W.G., and Ting, C.S., "Studies of Varying Behaviors for Fluid Over a Checking Valve," J. Taiwan Water Conserv., Vol. 42, No. 4, pp.67-73 (1994).

13. Kerh, T., Lee, J.J., and Wellford, L.C., "Transient FluidStructure Interaction in a Control Valve," J. Fluids Eng.--ASME, Vol. 119, pp.354-359 (1997).

14. Kerh, T., "Application of Galerkin Time Scheme to Investigate Unsteady Flow Around an Inclined Plate," Int. J. Modelling Simula., Vol.20, No. 1, pp. 69-78 (2000).

15. Kerh, T., "Computing of Viscous Fluid in a Backflow Preventer with an Oscillating Boundary," Int. J. Fluid Mech. Res., Vol. 29, No. 2, pp. 1-15 (2002).

16. King, M.J., Carden, J., David, T., and Fisher, J., “A Three-Dimensional Time-Dependent Analysis of Flow Through a Bileaflet Mechanical Heart Valve Comparison Experimental and Numerical Results," J. Biomech., Vol. 29, No. 5, pp. 609-618 (1996).

17. Lim, W.L., Chew, Y.T., Chew, T.C., and Low, H.T., "Steady Flow Dynamics of Prosthetic Aortic Heart Valves a Comparative Evaluation with PIV Techniques, "J. Biomech., Vol. 31, pp. 411-421 (1998).

18. Liou, T.M. and Liao, C.C., "Flow Fields in Lateral Aneurysm Models Arising From Parent Vessels with Different Curvatures Using PTV," Exp. Fluids, Vol. 23, pp. 288-298 (1997).
19. Muste, M., Fujita, I. and Kruger, A., "Experimental Comparison of Two Laser-Based Velocimeters for Flows with Alluvial Sand," Exp. Fluids, Vol. 24, pp.273 -284 (1998).

20. Oculus-F/64, "Device Driver," Version 1.30, Coreco Inc., USA (1994).

21. Oculus-F/64, "Frame Grabber User Manual," Edition 1.0, Revision 2, Coreco Inc., USA (1994).

22. Rehm, J.E. and Clemens, N.T., "An Improved Method for Enhancing the Resolution of Conventional DoubleExposure Single-Frame Particle Image Velocimetry," Exp. Fluids, Vol. 26, pp. $497-504$ (1999).

23. Reif, T.H., "A Numerical Analysis of the Backflow Between the Leaflets of a St. Jude Medical Cardiac Valve Prosthesis," J. Biomech., Vol. 24, pp.733-741 (1991).

24. Senthilnathan, V., Treasure, T., Grunkemeier, G. and Starr, A., "Heart Valves: Which is the Best Choice?" Cardiovasc. Surg., Vol. 7, No. 4, pp. 393-397 (1999).

25. Tecplot, "User Manual," Version 9, Amtec Engineering, Inc., USA (2002).

26. Tsen, F.G., "Digitalized Flow Visualization - Application to the Investigation of low Patterns of Natural Convection in an Enclosure," Master Thesis, Graduate Institute of Applied Mechanics, National Taiwan University (1991).

27. Wung, T.S., "Color-Coded Particle Tracking Velocimetry for 2-D and 3-D Fluid Flow Measurement," Proceedings of The International Workshop on PIV - Fukui'95, Japan, pp. 71-78 (1995).

28. Wung, T.S. and Lu, J.Y., "Three-Dimensional Positioning of a Particle from Stereo Picture Imaging by one Video Camera," J. Chin. Soc. Mech. Eng., Vol. 16, No. 2, pp. 141-153 (1995).

29. Young, D.F., Munson, B.R., and Okiishi, T.H., “A Brief Introduction to Fluid Mechanics," John Wiley and Sons, Inc., New York, pp. 324-332 (1997). 